\title{
UTERINE ARTERY EMBOLIZATION AS AN EFFICACIOUS STAND- ALONE TECHNIQUE IN MANAGEMENT OF SYMPTOMATIC UTERINE LEIOMYOMATA
}

\author{
Mounir Sobhy Guirguis; Mai Moutaz Rahmy; Amr Mahmoud Abdelsamad and \\ Wessam Sherin Shokry
}

Department of Radio diagnosis, Faculty of Medicine, Ain Shams University, Cairo, Egypt.

Corresponding author Mai Moutaz Rahmy

Mobile: (+2) 01066476285

E.mail:

supermayoya@hotmail.com

Received: $13 / 7 / 2021$

Accepted: 9/8/2021

Online ISSN: 2735-3540

\begin{abstract}
:
Background: Uterine artery embolization is now evolving to be the first-line therapy for symptomatic uterine leiomyoma alongside elder conventional alternatives comprising hysterectomy and myomectomy whether surgical or endoscopic. Many clinical trials have confronted UAE and surgical therapies as regard safety and efficacy in treatment of symptomatic leiomyomas where UAE exhibited comparable short and mid-term clinical outcomes, encompassing symptomatic amelioration, better quality of life and psychological satisfaction benefiting from uterine preservation.
\end{abstract}

Aim of the Work: to emphasize the role of the trans-catheter uterine artery embolization as an effective stand-alone technique in treating the symptoms attributable to uterine leiomyomata.

Patients and Methods: This study included 30 patients with uterine fibroids referred from gynecology clinic to interventional radiology unit to be counseled for UAE.

Results: Follow-up symptomatic assessment was done for all patients at 1 and 3 months. Most patients confirmed symptomatic amelioration at 1 months persisting or continue at 3-month follow-up visit. Among 21 patients presented with bleeding, 90.5\% of patients reported symptomatic improvement at 1 month post-procedurally continued to increase to reach $95.2 \%$ at 3 months follow-up visit, however, no significant symptomatic change was noted between 3, 6 months follow up. Fourteen patients complained from pressure symptoms. At 1-month follow-up, $71.44 \%$ of patients stated improvement, $14.29 \%$ had stationary course with similar percentage exhibited slightly worse response. Contrariwise, $85.71 \%$ of patients reported symptomatic amelioration at 3 month and $14.3 \%$ had no change. Indifferent from bleeding, a highly statistically significant time improvement between 1, 3 months follow up is notable. Of 13 patients admitted with pelvic pain, $53.9 \%$ get improved, $30.77 \%$ showed no change, $15.38 \%$ gave slightly worse response in comparison to $84.7 \%, 7.69 \%, 7.69 \%$ at 1, 3 months follow-up clinical assessment visits. There is clinical reduction of pain between 1, 3 months however it didn't reach clinical significance. Among three patients presented with infertility, one gets pregnant at 3 months follow up with no significant change between 1, 3 months follow up. On MRI, the mean uterine volume was reduced by $30 \% 6$ months after treatment. Similarly, mean dominant leiomyoma volume was reduced by $44 \%$ after 6 months. There was highly statistically reduction of volume between 1 and 6 months follow up. 
Conclusion: Although limited by small sample size however we might conclude that UAE is associated with considerable cumulative reduction of leiomyoma volume that parallels patients' symptomatic amelioration and psychological satisfaction and should be considered among first lines of management of symptomatic leiomyoma particularly in child-bearing age.

Keywords: Uterine artery embolization; symptomatic uterine leiomyomata

\section{INTRODUCTION:}

Uterine leiomyomata also known as fibroids are the most considerably occurring tumor that involve reproductive tract in a premenopausal women. The peak incidence has been demonstrated in women in childbearing age with higher risk multiplied by three among black more than white women ${ }^{(1)}$.

Encountered as a benign asymptomatic pathology, nevertheless, uterine fibroids may present with variable disabling symptoms that interfere with normal life activities. Uterine Leiomyomata may give rise to heavy menstrual bleeding with subsequent iron deficiency anemia, pelvic pain, discomfort, pressure - related symptoms as well as infertility issues ${ }^{(2)}$.

Management of symptomatic uterine leiomyomata have been traditionally encompasses diverse treatments ranging from medical treatment up to hysterectomy ${ }^{(3)}$. Symptomatic fibroids are used to be treated with hysterectomy whenever having unsatisfactory outcome with medical treatments. Hysterectomy is associated with significant morbidities, 10$15 \%$ complication rates as well lengthy hospital stay and prolonged recovery time ${ }^{(4)}$.

Patients' desire to keep their uterus have prompted the development of uterus sparing alternatives as laparoscopic, hysteroscopic myomectomies ${ }^{(5)}$. Presence of multiple fibroids with variable size, location adding to associated pain, high post-operative morbidity as well as symptoms recurrence ( $25 \%$ of patients] have made myomectomy not feasible in all circumstances ${ }^{(\boldsymbol{(})}$. Moreover; patients who have undergone myomectomy are also at risk of developing recurrent symptomatic fibroids. The reported incidence varies between $10 \%$ and $62 \%$.

UAE technique initially introduced to control post-partum hemorrhage, was used successfully in the early nineties, on a preoperative basis during the 3-10 days prior to myomectomy procedure to facilitate surgical removal, reduce complications related to intra-operative bleeding ${ }^{(7)}$.

In 1995; UAE was proposed as an alternative uterine -sparing technique for treating symptomatic leiomyomata ${ }^{(8)}$. Multiple subsequent published studies have discussed its efficacy in the short, mid-term and long term with lower notable complication rate and reduced hospital stay ${ }^{(9)}$.

\section{PATIENTS AND METHODS:}

Our study is a prospective cohort study that was held during the period from April 2018 till September 2020, in radiodiagnosis department, Faculty of medicine, Ain shams University. It included 30 patients referred from gynecology clinic along with their ultrasonographic reports to interventional radiology unit to be counseled for UAE.

Study population: Patients subjected to the study according to the following criteria:

Inclusion criteria: Any women who had uterine leiomyoma (s) as evident on sonography with heavy menstrual bleeding/menorrhagia, or pressure symptoms related to leiomyoma mass effect, 
or infertility attributable to leiomyoma with no other definable cause and pelvic pain.

Exclusion criteria: Pregnancy, postmenopausal bleeding attributable to coexistent pathology other than leiomyoma, pedunculated sub mucosal or cervical leiomyomas, non-richly vascularized/ nonenhancing/ necrotic leiomyoma on preassessment MRI, small leiomyoma less than $1 \mathrm{~cm}$, renal dysfunction with high serum Creatinine reaching up $>2 \mathrm{mg} / \mathrm{dl}$, history of life-threatening contrast hypersensitivity, coagulopathies and blood disorders, and bacteremia at time of endovascular treatment.

\section{Technique of Uterine artery embolization (UAE):}

\section{Patient preparation:}

Proper history taking, full clinical assessment, pre-procedural assessment of renal function via blood urea \& serum Creatinine, Pelvic magnetic resonance imaging (MRI) with contrast aiming to confirm leiomyoma diagnosis, determine their location, and exclude other co-existent pathologies like adenomyosis as well as to assess their vascularity which gives a clue about treatment feasibility. The uterine, dominant leiomyoma volumes were calculated in $\mathrm{cm}^{3}$ pre-procedural as a base line and 6 months post-procedural using the formula for a simplified prolate ellipse ( $\mathrm{L} \mathrm{X}$ W X D X .542), administration of an antecubital intravenous catheter, and detailed explanation of imaging procedure with obtaining written informed consent.

\section{Technique of embolization:}

The procedure was done under local anesthesia with fluoroscopic control using monoplane Toshiba unit. A 5French arterial introducer (Radifocus, TERUMO) was placed at right femoral artery in 16 patients whilst bilateral femoral puncture was done in 14 patients.
Double 5F Cobra $_{2}$ catheter (Cordis) over a $0.35 \mathrm{~F}$ hydrophilic guide wire (Radifocus; TERUMO) was navigated into both uterine arteries when adopting bilateral femoral puncture. Upon, single puncture technique; selective catheterization of the contralateral (left) uterine artery was implemented first followed by formation of a Waltman loop and withdrawal of the catheter to select the ipsilateral (right) uterine artery till its transverse portion.

Under fluoroscopy, injection of contrast was done, to examine vessel configuration, to exclude presence of vasospasm or dissection and to determine number of leiomyomas, characterize their angiographic pattern and feeding arteries.

The embolization of both uterine arteries was done utilizing Embosphere particles (Biosphere Medical) 500- $700 \mu \mathrm{m}$, $700-900 \mu \mathrm{m}$ up to $900-1200 \mu \mathrm{m}$ followed by torpedoes of gel foam in 4 patients in order to achieve complete flow cessation. Unilateral UAE embolization was performed in one patient due to technical difficulties as a result of iatrogenic arterial spasm. The technical end point was stasis in the main uterine artery for not less than 10 cardiac beats.

\section{Post - procedural precautions/ medications}

- Puncture site hemostasis was obtained by manual compression for 15 consecutive minutes.

- Strict bed rest for six hours to minimize the risk of hematoma.

- Analgesia and prophylactic broadspectrum antibiotics to control postprocedural pain, avoid superimposed infection.

\section{Risks and complications:}

Uterine artery Embolization is generally a safe procedure with low risk of major or life-threatening complications. Ovarian failure and ischemia of other pelvic organs 
are the most serious complications that could result from non-target embolization.

However, the most common transient complications associated with embolization are: Pelvic Pain and fever, puncture site complications (e.g., hematomas) are also fairly-frequent and usually are of no clinical consequence, Signs of infection that may develop in the days following removal of the sheath or even after discharge.

\section{Treatment in cases of risks and complications:}

Analgesia; NSAIDs, broad spectrum antibiotics, IV atropine, IV fluids as well as oxygen via a mask should be administered and the bed tilted to head low position in case of vasovagal reaction.

\section{Procedural outcome:}

To assess post-procedural technical success of UAE; all patients were subjected to pelvic MRI with contrast 6 months after the procedure to evaluate reduction in uterine and dominant leiomyoma volumes as well as necessity for re-interventions via repeated sessions of UAE or subsequent myomectomies and hysterectomies. Uterine leiomyoma shrinkage volume inferred from pre and post-operative MRI scans will be correlated with symptomatic improvement/ deterioration or stability.

On clinical basis, Follow-up of the patients' symptoms were monitored 1 and 3 months after discharge by verbal questionnaire. The bleeding, pressure and pain symptoms improvements were graded as follow: (Significantly worse, 1 - Slightly worse; 2 - Stationary; 3 - Slightly improved; 4 - Significantly improved; 5). Patient psychological satisfaction at these follow-ups visits was also rated as: Satisfied (3), neither (2), dissatisfied (1).

To estimate the physical progress, patients' data as regard length of hospital stay, days till return to normal activity, reported complications were assembled. According to Society of Interventional Radiology (SIR) standards; complications were subdivided into: (1) Major complication that necessitates longer hospital stay than expected or that leaves long-lasting adverse effect. (2) Minor complications that can be self - limiting or solved on a day case admission.

Descriptive data obtained was the procedure parameters, uterine and leiomyoma volume changes, and questionnaire responses. As baseline parameters, the patient's age, most annoying symptom, the uterine and dominant fibroid volumes, sum of leiomyomata, and site of dominant leiomyoma were all gathered.

\section{Statistical analysis:}

Data were collected, revised, coded and entered to the Statistical Package for Social Science (IBM SPSS) version 22. The quantitative data were presented as mean, standard deviations and ranges when parametric. Also qualitative variables were presented as number and percentages. The comparison between groups with qualitative data was done by using Chi-square test. The comparison between two paired groups with quantitative data and parametric distribution were done by using Paired t-test. The confidence interval was set to $95 \%$ and the margin of error accepted was set to $5 \%$.

\section{Study Ethics:}

Full secrecy is granted and only the main supervisor will have access to the participant's data and results. The participants signed a written consent and were fully informed of the results of his/her examination. Any participant does not have to take part in this research if he/she refuses to. Any participant may also stop participating at any time. In case of refusal the participant will have his/her examination done.

\section{RESULTS:}

A total of 30 females with mean age of 34.2 (31-45 ys) presented with uterine leiomyomata where menstrual irregularities whether heavy or prolonged bleeding were the most common clinical presentation 
amounting to $70 \%$ in our study (21 patients). Thirteen patients $(43.3 \%)$ complained from persistent intolerable pelvic pain in form of heaviness. Whilst 14 patients $(46.7 \%)$ experienced pressure symptoms varied between dysuria and constipation, 3 patients $(10 \%)$ came seeking fertility after excluding all possible primary causes of infertility with adequate partners' semen analysis (Diagram 1).

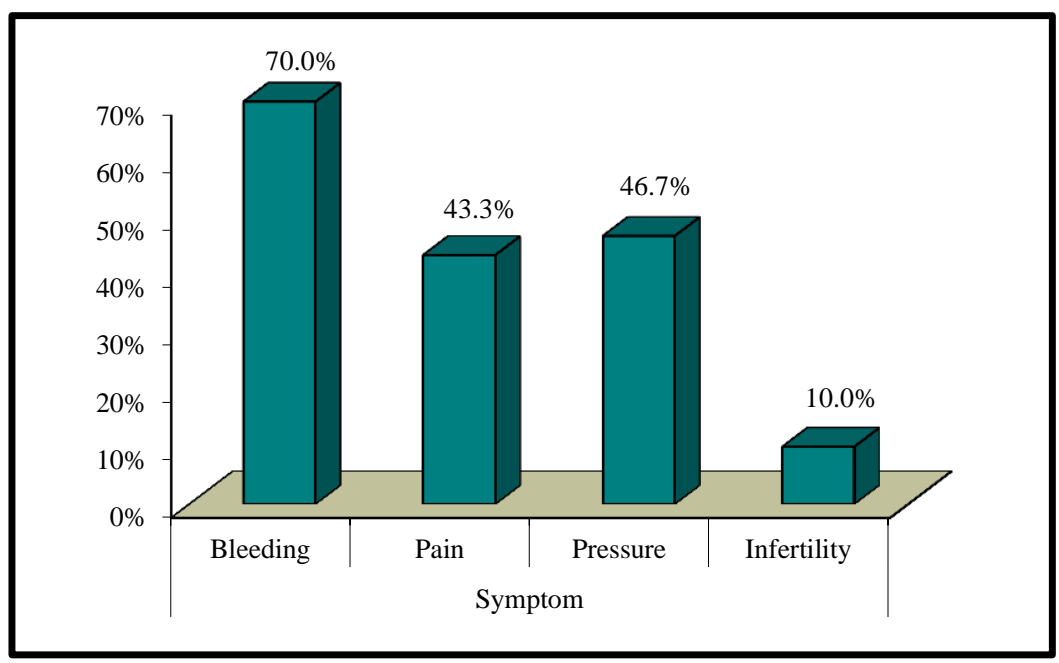

Diagram (1): Patients categorized according to clinical presentation.

Preprocedural MRI revealed 7 patients $(23.33 \%)$ with solitary large leiomyoma whilst majority of patients $(56.67 \%, \mathrm{n}=17)$ harbored between 2-5 leiomyomas. More than 5 leiomyomas were seen in $20 \%$ of patients $(\mathrm{n}=6)$ (Diagram 2).

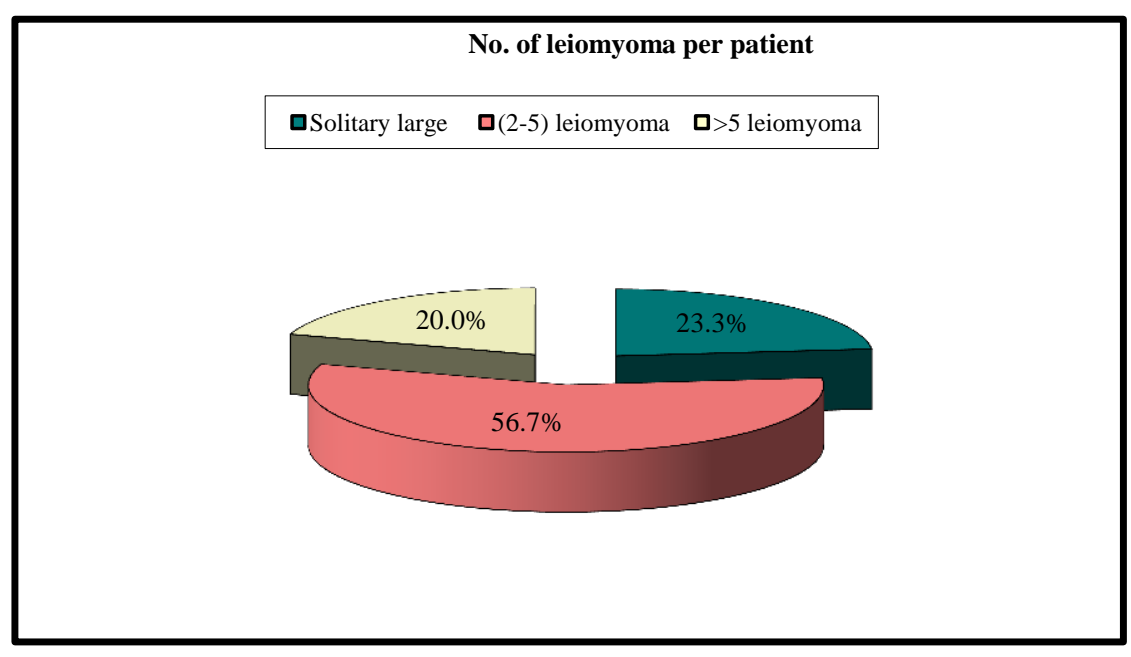

Diagram (2): Number of leiomyomas in our study per patient.

With respect to dominating myomas location in relation to uterine wall layers: $26.67 \%(n=8)$ of myomas were depicted submucosally whereas the preponderance
$53.33 \% \quad(n=16)$ were intramural. Subserosal leiomyomas were represented only in $20 \%(\mathrm{n}=6)$ (Diagram 3). 


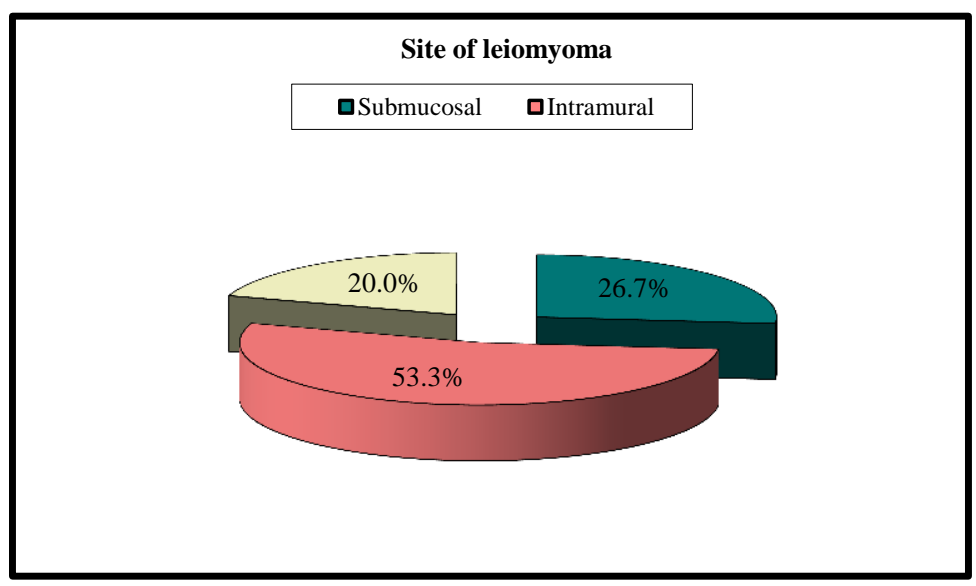

Diagram (3): Location of leiomyomas in relation to uterine layers.

Preprocedural analysis of MRI showed $1165 \mathrm{~cm}^{3}$ ) and 279.6 (Range: 77-486 $\mathrm{cm}^{3}$ ) mean of uterine as well as dominant leiomyoma volumes of 783.33 (range: 333-
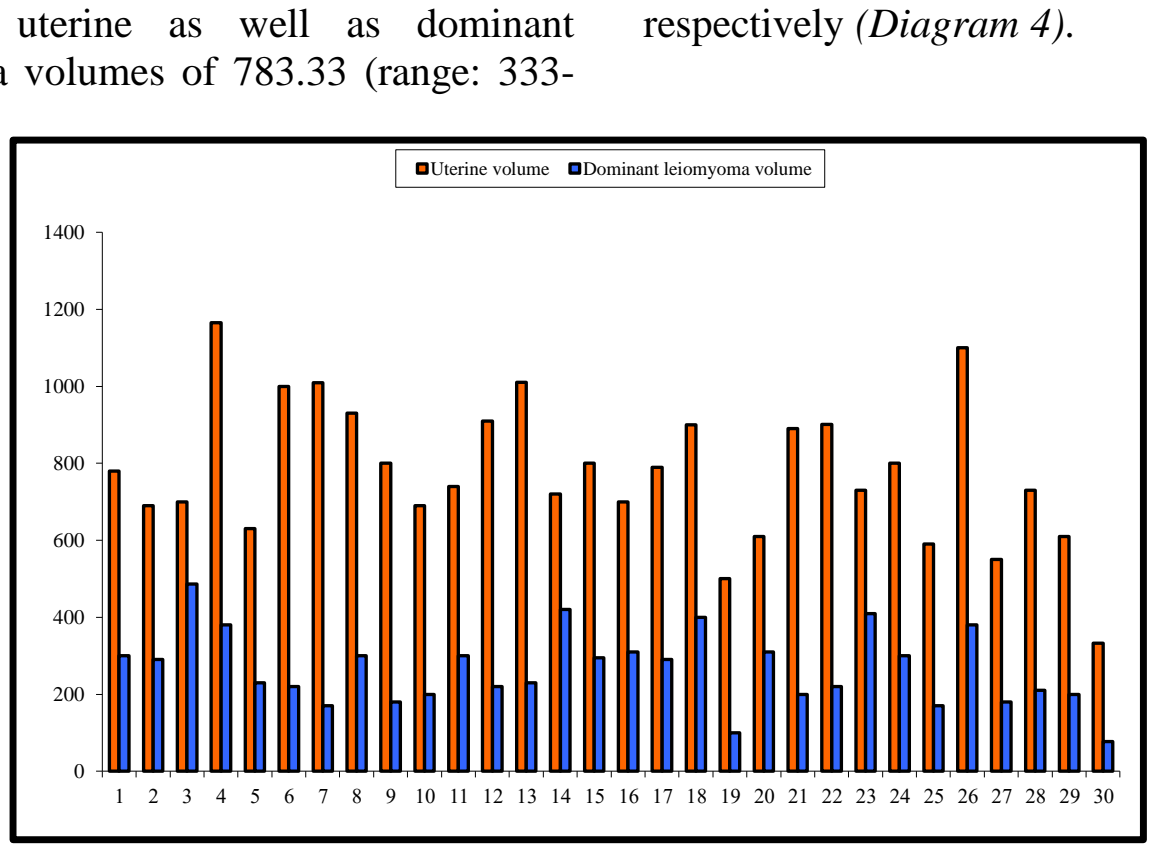

Diagram (4): Uterine and dominant leiomyoma volumes as measured on preprocedural MRI.

Uterine artery embolization was technically successful performing embolization via both uterine arteries in twenty-nine patients. Nonetheless, unresolving arterial spasm prohibited left uterine artery catheterization, hence only unilateral approach was done.

Average length of hospital stay was 8 (range: 6-24) hours where majority of patients were discharged at the same/ next day of the procedure. Overnight (more than 24-hour) admission was necessary only in $13.33 \%$ of patients. Seven (range: 5-10) days were the average for patients to return to normal activity following the procedure.

No major complications were noted in our study with no necessity for further gynecologic interventions like myomectomy or hysterectomy. Only 5 patients had minor complications, two of them experienced intolerable persistent pelvic pain necessitating night admission, however it was controlled afterwards by analgesics and non-steroidal anti-inflammatory agents. One patient had post-embolization syndrome manifested by low grade fever, pain, nausea 
and vomiting but was resolved one week after aided by analgesics and IM antibiotics for 5 days. Immediate post-procedural complications were depicted in two patients having femoral hematoma following sheath removal yet was localized and controlled after adequate compression for 30 minutes.

Follow-up symptomatic assessment was done for all patients at 1,3 months. Most patients confirmed symptomatic amelioration at 1 month persisting or continue at 3months follow-up visit. Among 21 patients presented with bleeding, $90.5 \%$ of patients reported symptomatic improvement at 1 month post-procedurally continued to increase to reach $95.2 \%$ at 3 months followup visit, however, no significant symptomtic change was noted between 1, 3 months follow up $(\mathrm{p}=0.529)$ (Table 1).

Fourteen patients complained from pressure symptoms. At 1-month follow-up, $71.44 \%$ of patients stated improvement,
$14.28 \%$ had stationary course with similar percentage exhibited slightly worse response. Contrariwise, $85.71 \%$ of patients reported symptomatic amelioration at 3 months and $14.3 \%$ had no change. Indifferent from bleeding, a highly statistically significant time improvement between pre procedure and 1, 3 months follow up is notable $(\mathrm{p}=0.005)$ (Table 1).

Of 13 patients admitted with pelvic pain, 53.8\% get improved, 30.8\% showed no change, $15.4 \%$ gave slightly worse response in comparison to $84.6 \%, 7.7 \%, 7.7 \%$ at 1,3 months follow-up clinical assessment visits. There was clinical reduction of pain between 1, 3 months however it didn't reach clinical significance $(\mathrm{P}=0.129)$.

Among three patients presented with infertility, one gets pregnant at 3 months follow up with no significant change between 1,3 months follow up $(\mathrm{P}=0.313)$ (Table 1).

Table (1): Post-procedural presenting symptoms as perceived 1, 3 months follow-up visits.

\begin{tabular}{|c|c|c|c|c|c|c|c|c|}
\hline & \multicolumn{2}{|c|}{1 month } & \multicolumn{2}{|c|}{3 month } & \multirow[t]{2}{*}{ Test value* } & \multirow[t]{2}{*}{ P-value } & \multirow[t]{2}{*}{ Sig. } \\
\hline & & No. & $\%$ & No. & $\%$ & & & \\
\hline \multirow{2}{*}{ Bleeding } & Yes & 2 & $6.7 \%$ & 1 & $3.3 \%$ & \multirow[t]{2}{*}{0.351} & \multirow[t]{2}{*}{0.554} & \multirow[t]{2}{*}{$\mathrm{NS}$} \\
\hline & No & 28 & $93.3 \%$ & 29 & $96.7 \%$ & & & \\
\hline \multirow[t]{2}{*}{ Pressure } & Yes & 4 & $13.3 \%$ & 2 & $6.7 \%$ & \multirow[t]{2}{*}{0.741} & \multirow[t]{2}{*}{0.389} & \multirow{2}{*}{ NS } \\
\hline & No & 26 & $86.7 \%$ & 28 & $93.3 \%$ & & & \\
\hline \multirow[t]{2}{*}{ Pain } & Yes & 6 & $20.0 \%$ & 2 & $6.7 \%$ & \multirow[t]{2}{*}{2.308} & \multirow[t]{2}{*}{0.129} & \multirow[t]{2}{*}{ NS } \\
\hline & No & 24 & $80.0 \%$ & 28 & $93.3 \%$ & & & \\
\hline \multirow{2}{*}{ Infertility } & Yes & 3 & $10.0 \%$ & 2 & $6.7 \%$ & \multirow[t]{2}{*}{0.218} & \multirow[t]{2}{*}{0.641} & \multirow[t]{2}{*}{$\mathrm{NS}$} \\
\hline & No & 27 & $90.0 \%$ & 28 & $93.3 \%$ & & & \\
\hline
\end{tabular}

P-value $>0.05$ : Non significant (NS); P-value <0.05: Significant (S); P-value $<0.01$ : highly significant (HS) *:Chi-square test

Patient satisfaction was analogous to improvement on clinical scale. Nineteen patients $(63.3 \%)$ of patients were satisfied at
1 month follow up which increased up to 25 patients $(83.3 \%)$ at 3 months (Diagram 5$)$. 


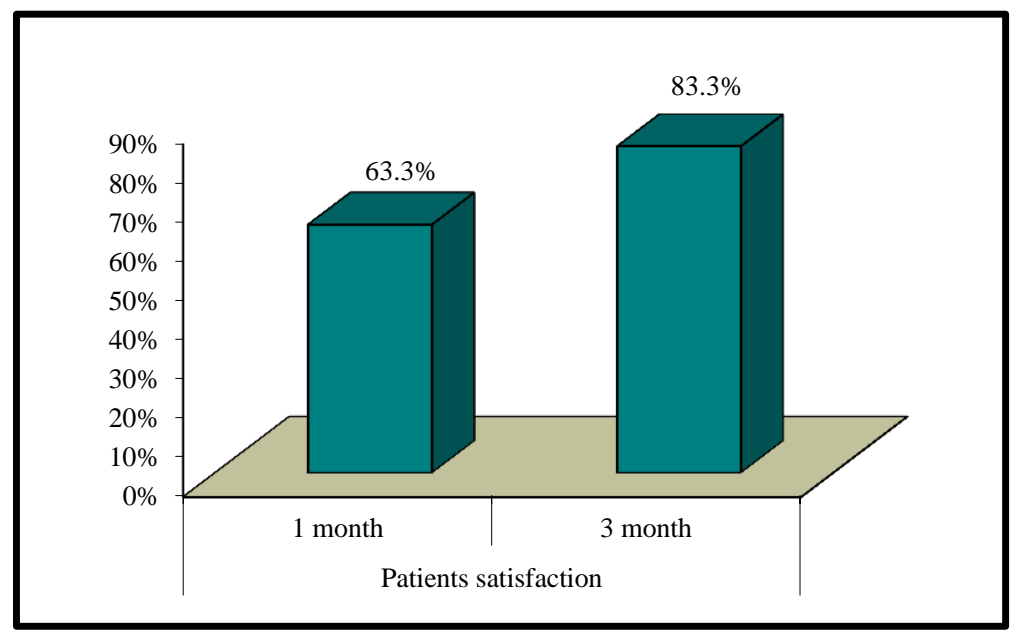

Diagram (5): Post-procedural patient satisfaction as perceived 1, 3 months follow-up visits.

On MRI, the mean uterine volume was reduced by $30 \%$ (standard deviation [SD] 0.03) 6 months after treatment. Similarly, mean dominant leiomyoma volume was reduced by $44 \%$ (SD 0.06) after 6 months. There was highly statistically reduction of volume between 1 and 6 months follow up ( $\mathrm{P}$ value $<0.001)$ (Table 2).

Table (2): Pre and 6 months post-procedural patient uterine volume.

\begin{tabular}{|l|l|c|}
\hline \multicolumn{2}{|c|}{ Uterine volume } & No. $=30$ \\
\hline \multirow{2}{*}{ Pre } & Mean \pm SD & $783.33 \pm 183.14$ \\
\cline { 2 - 3 } & Range & $333-1165$ \\
\hline \multirow{3}{*}{ month } & Mean \pm SD & $672.20 \pm 182.10$ \\
\cline { 2 - 3 } & Range & $296-1075$ \\
\hline \multirow{2}{*}{ change } & Mean \pm SD & $13.93 \pm 6.24$ \\
\cline { 2 - 3 } & Range & $2.56-29.51$ \\
\hline Paired t-test & 13.212 \\
\hline P-value & $<0.001(\mathrm{HS})$ \\
\hline
\end{tabular}

Post-procedural subtraction $\quad$ MRI $\quad 5.6 \pm 0.5$ SI in contrast to $133.4 \pm 18.3 \mathrm{SI}$ revealed mean myoma enhancement to be prior to embolization.

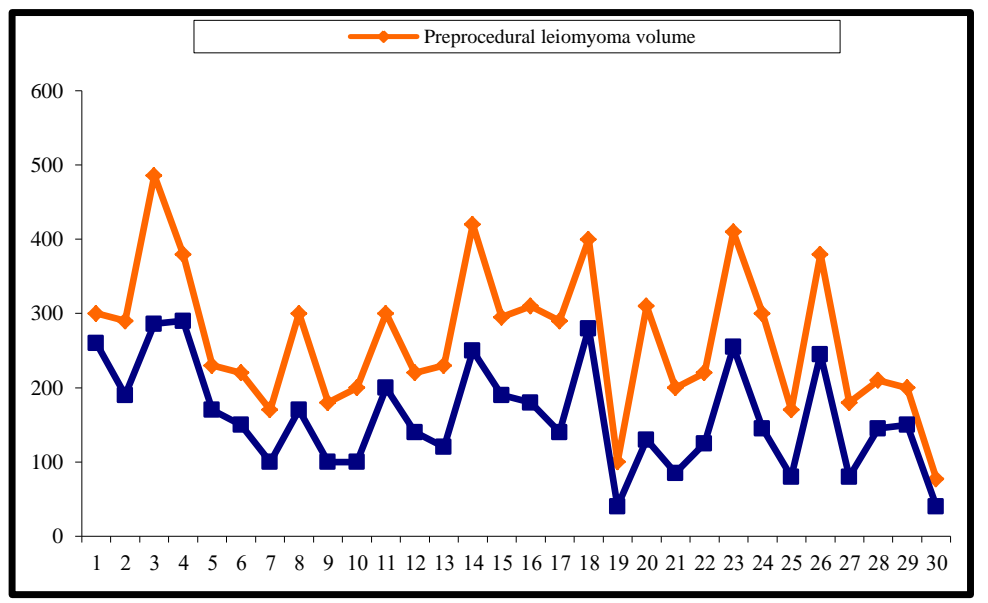

Diagram (6): Pre-procedural and 6 months follow up leiomyomata volumes following UAE. 


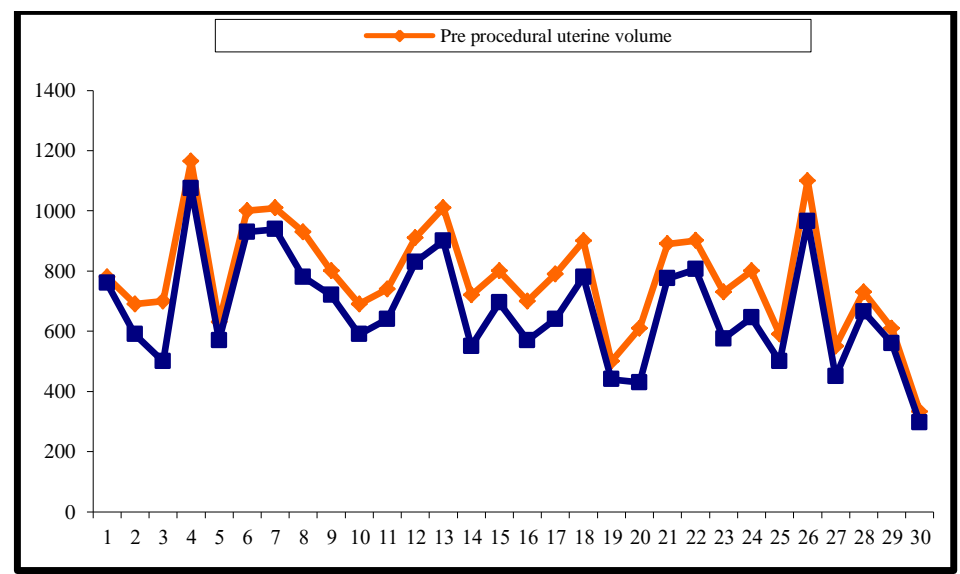

Diagram (7): Pre-procedural and 6 months follow up uterine volumes as measured on MRI.
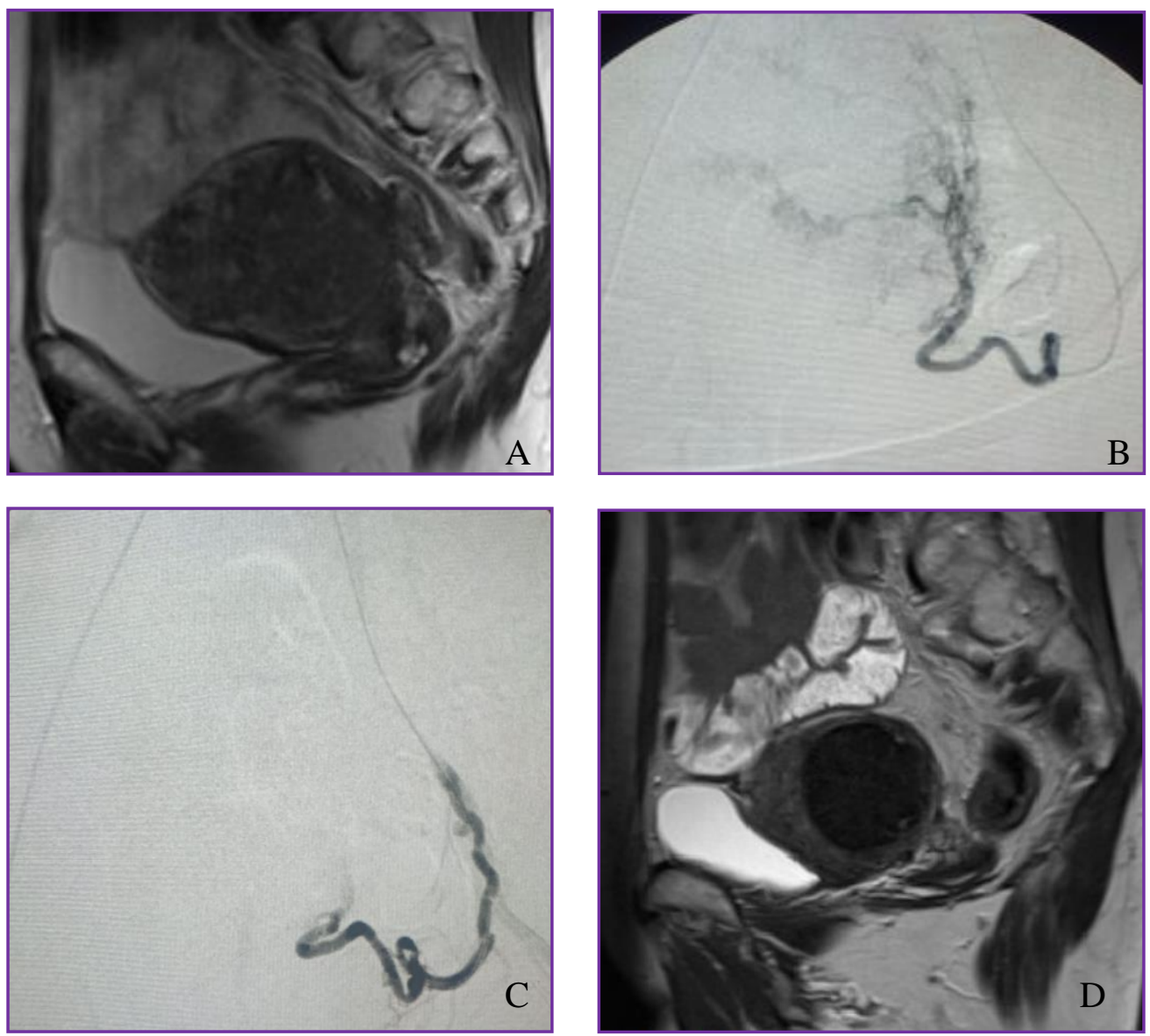

Fig. (1): A) Pre-embolization Sagittal MRI revealed a solitary intramural homogeneous leiomyoma of average volume $294 \mathrm{~mm}^{3}$. B) Digital subtraction angiography (DSA) revealed vascular leiomyoma blush before embolization (B) and after embolization (C). D) Post-embolization sagittal T2W revealed a significant reduction in leiomyoma volume concomitant with symptomatic improvement. 

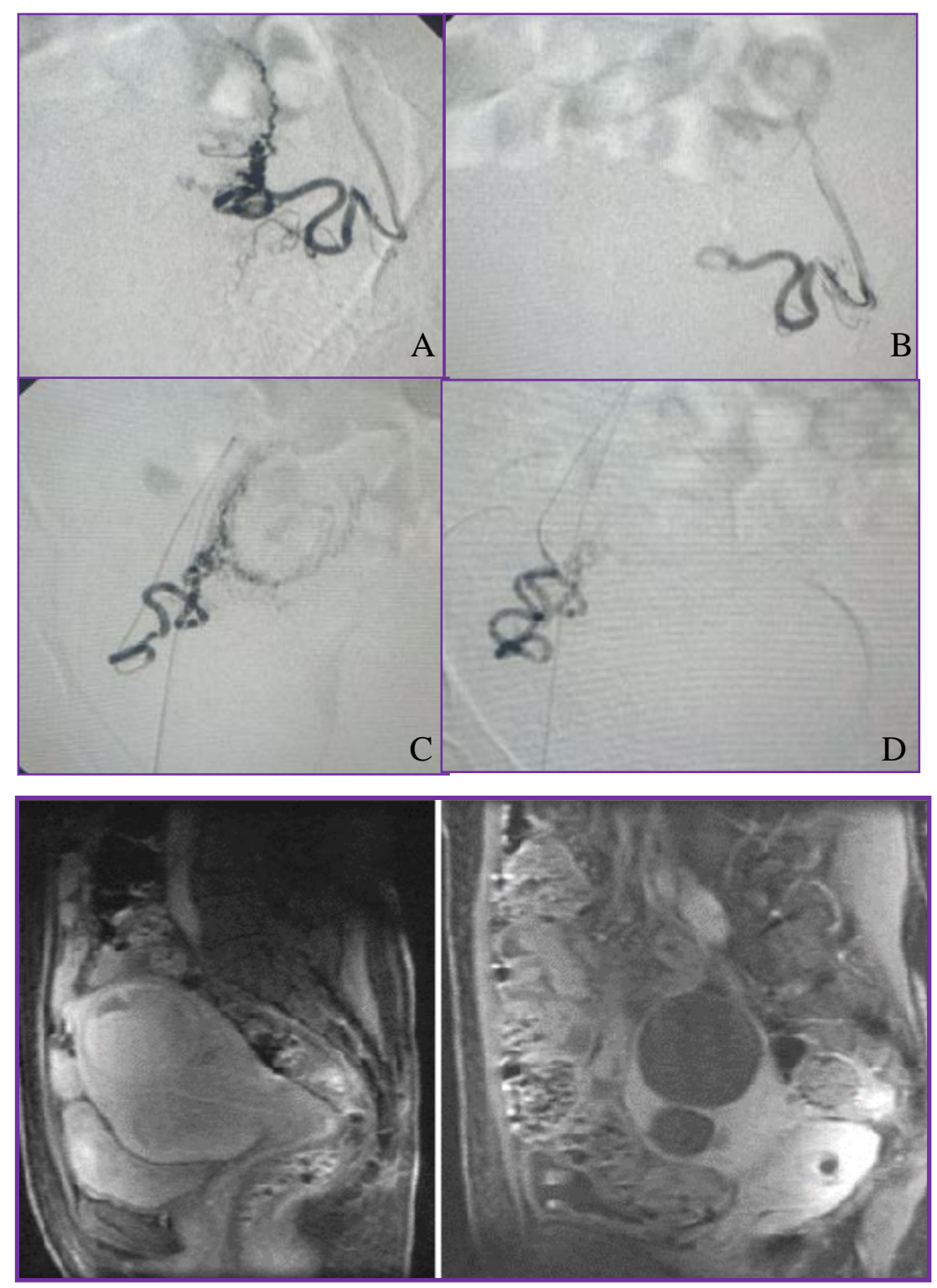

Fig. (2): Digital subtraction angiography (DSA) revealed two uterine leiomyomas supplied by both uterine arteries (A). Embolization was done utilizing Embosphere particles of 700-900M followed by gel foam torpedoes $(B)$. Axial T1W Fat saturation MRI after gadolinium contrast injection demonstrated two enhancing leiomyomas of average diameters $77,233 \mathrm{~mm}^{3}$ (D). Post-embolization axial T1W Fat saturation MRI post-gadolinium contrast injection depicted a relative reduction in leiomyoma volume concomitant with lack of contrast enhancement that signify total infarction and subsequent liquefaction $(\mathrm{C})$.

\section{DISCUSSION:}

Since Ravina et al. reported their results in $(\mathbf{1 9 9 5})^{(\mathbf{1 0 )}}$; uterine artery embolization has received widespread attention as a non surgical alternative in the treatment of symptomatic uterine fibroids. However, because of the pace of development, confusion has aroused over the proper indications of various procedures and the relative roles of percutaneous and conventional surgical procedures. It is important to remember that for any given patient, alternative treatment may be valid, 
and preferred for sound clinical reasons. In addition, certain surgical or percutaneous procedures require highly trained and unusually skilled physicians.

Abnormal uterine bleeding is common in patients who are between 35 and 49 years of age ${ }^{(11)}$ and occurring in $35-60 \%$ of women who are older than 40 years ${ }^{(12)}$.

As stated by Brunereau et al. ${ }^{(13)}$ and Levy and Spies ${ }^{(14)}$ that abnormal uterine bleeding may be due to many causes .Uterine leiomyoma is infront of these causes .Bleeding may be accompanied by pelvic pain, heaviness, discomfort and even anemia. The main symptom in our patients was abnormal bleeding in 21 patients accounting for $70 \%$ of the cases.

In a meta-analysis by Torre et al. ${ }^{(6)}$ at 1-year follow-up rate of symptomatic improvement was 87.5 (84.7-90.0) for heavy menstrual bleeding. Our results for menorrhagia mirror those results where $96.7 \%$ of patients reported stable improvement of menstrual bleeding after 3 months follow up.

Torre et al. ${ }^{(\boldsymbol{( 6 )}}$ also stated at 1-year follow-up rate of symptomatic improvement was $86.6 \%$ (82.2-90.5) for pressure-related symptoms. Our results reported that $93.3 \%$ of patients with pelvic pressure related symptoms improved at 3 months follow up.

Torre et al. ${ }^{(6)}$ also reported at 1-year follow-up, rate of pain improvement was $77.5 \%$ (73.8-80.9). Our results for pain at 1 month follow up were only $53.8 \%$ of patients presented with pelvic pain reported pain resolution which is inferior to that stated in the literature which may be attributable to consistent complete occlusion of both uterine arteries which may induce relatively severe pain comparable to incomplete embolization as stated by Marshburn et $\boldsymbol{a l} .{ }^{(15)}$ Although there was a notable time interval cumulative betterment of pain to reach $84.6 \%$ of patients at 3 months follow-up however it didn't reach statistical significance.

Patient satisfaction results were analogous to symptomatic improvement (bleeding and pain) as $63.3 \%, 83.3 \%$ of patient gave satisfactory response at 1, 3 months following the procedure respectively.

Despite that normal uncomplicated pregnancy can occur after UAE, it should be offered for those refusing surgical interventions like the three patients included in our study, from which one got pregnant at 3 months follow up. Future prospective studies on larger populations are in need to fully evaluate UAE procedure impact on fertility rates, placenta as well as uterine issues.

Ultrasound is considered the primary imaging modality used to evaluate women with gynecological symptoms suspected to be attributable to leiomyoma on physical assessment. Nonetheless, MR imaging is considered the gold standard modality of choice for potential UAE candidates. It could precisely affirm patient eligibility for the procedure after excluding mimic causes of abnormal uterine bleeding as well as pelvic bleeding. Moreover, MRI is beneficial in predicting UAE outcome and determining potential risks for complications ${ }^{(16)}$.

Although associated with relatively high cost, MRI is a non-invasive procedure that could precisely confirm uterine leiomyoma diagnosis while impacting patients' management by decreasing number of superfluous surgeries with analogous reduction in healthcare expenditures as stated by Liapi et al. ${ }^{(17)}$. In concurrence with Orazio et al. ${ }^{(18)}$, accurate uterine leiomyomas detection and localization might lead to alteration of planned therapy apart from ultrasound or clinical assessment.

A notable contradiction between ultrasound and MRI assessment capability prior to UAE in term of uterine volume, number and location of leiomyomas was 
obvious in Spielmann et al. ${ }^{(19)}$ who studied 49 patients and concluded that MRI affords complementary information to clinical and sonographic assessment. MRI altered clinical management in 11 patients, as four patients were excluded from UAE, while seven women underwent embolization after being deemed unsuitable candidates for UAE at primary US evaluation.

Pelvic MRI dedicated to characterizing leiomyomas should include T2-weighted images in at least two orthogonal planes (generally axial and sagittal) to define the uterine axis and determine leiomyomas location as set by Das et al. ${ }^{(20)}$ Additionally, protocol should adjoin axial T1-weighted images, opposed-phase GRE or fast spinecho (FSE), to delineate leiomyomas relationship to uterine layers and adnexa and differentiate blood and fat signals facilitating diagnosis of concurrent uterine and adnexal disease. In line with our protocol, threedimensional dynamic non-enhanced and contrast-enhanced fat-suppressed GRE images were taken to portray leiomyoma extension, enhancement properties and identify parasitized ovarian vessels if existent.

Providing valuable prognostic information regarding potential UAE technical and clinical efficacy, preprocedural MRI assessment should report and analyze number, location, size and stalk parameters (In pedunculated type) as well as contrast enhancement of leiomyomas.

Highest proportion of patients in our study presented with 2 leiomyomas whilst $23.33 \%(n=7)$ of patients came with solitary large leiomyoma. Meanwhile $20 \%$ of patients presented with more than 5 leiomyomas. In relation to uterine layers, Intramural leiomyomas represent the highest incidence whilst sub-serosal constitute the lowest proportion. Average uterine as well as dominant leiomyoma volumes were 776.93 and $265.93 \mathrm{~cm}^{3}$ respectively.
Leiomyomas at-risk encompassing intracavitary submucosal, cervical or pedunculated narrow-stalked sub-serosal types should be depicted cautiously at MRI prior to UAE as demonstrated by Cura et $\boldsymbol{a l}^{(21)}$. Although the latter is considered a relative contraindication for UAE for fear of possible stalk necrosis and detachment which might predispose to persistent pelvic pain or peritonitis. Nonetheless recently, stalk diameter of $2 \mathrm{~cm}$ or larger is considered the safety limit that might not be associated with major complication. Our study included 1 sub-serosal pedunculated leiomyoma yet wasn't of narrow-stalk type. Patient underwent UAE with remarkable size regression of the leiomyoma and no concomitant detachment or further complications.

Correspondingly, disconnection of pedunculated submucosal leiomyoma into uterine cavity might lead to transcervical expulsion of necrotic debris and possible cervical obstruction and superimposed infection necessitating surgical intervention. MRI could help predict the likelihood for submucosal leiomyoma to become intracavitary following UAE via contrasting leiomyoma volume relative to its endometrial interface. Verma et al. ${ }^{(22)}$ compared MR imaging features of thirty-nine submucosal leiomyomas before and after embolization. Ratio of largest endometrial interface (I) over largest leiomyoma dimension (D) was calculated pre-procedurally whilst change in leiomyoma location was monitored on postprocedural MRI. One-third of leiomyomas became intracavitary after UAE necessitating further intervention in 4 patients. Mean I/D ratio of these leiomyomas was significantly higher than the I/D ratio of leiomyomas that did not become intracavitary following UFE. Furthermore, the mean I/D ratio of the leiomyomas that became completely intracavitary after UFE was greater than those became partially intracavitary. 
Expulsion of leiomyomas or its fragments following UAE might occur in up to $10 \%$ of cases. Although multiparous women are more susceptible than nulliparous ones, yet it should be discussed with every single patient prior to intervention. Leiomyomas expulsion rate might be high as $50 \%$ when its volume is equal to or exceeds $66 \mathrm{~mL}$, corresponding to a diameter of nearly $5 \mathrm{~cm}$ as demonstrated by Zhang et $\boldsymbol{a l}^{(23)}$ Considering this risk particularly in view of huge volumes our myomas exhibit, pedunculated submucosal myomas were excluded from our study.

In concurrence with Deshmukh et al. ${ }^{(24)}$, leiomyomas enhancement is a powerful predictor for UAE response where surgical myomectomy or hysterectomy might be better alternative for non-enhancing devascularized leiomyoma particularly if the main complaint for the patient is related to pressure symptoms claiming that UAE is coupled with less volume reduction with concomitant less symptomatic clearance and patient unsatisfaction.

On the contrary, Deipolyi ${ }^{(25)}$ assumed that non-enhancing and presumably necrotic myomas still responds to UAE, in spite of their relative avascularity attributing it to myomatous avascular and hypoxic environment that is paradoxically associated with more proangiogenic factors.. Moreover, avidly enhancing tumors rapidly reduce in size shortly followed UAE but reach a plateau after 6 months, as opposed to less enhancing fibroids that continued to shrink after 6 months explaining why earlier studies with short term follow-up reported greater volume reduction in enhancing leiomyomas.

As regard post-UAE surveillance, MRI proved to beneficial in case of symptomatic persistence or worsening to evaluate leiomyoma volume shrinkage, location change and residual enhancement which might be attributable to parasitized supply that necessitates re-embolization. At 6 months follow-up MRI, leiomyomas routinely exhibit hypointense signal on $\mathrm{T} 2 \mathrm{~W}$ images with no enhancement following contrast material administration that is compatible with total infarction. However, variable signals may be noted on $\mathrm{T} 2 \mathrm{~W}$ along with T1-shortening effects of methemoglobin denoting hemorrhagic infarction. Occasionally, a minute amount of gas might be present intra-myomal which does not equate with a pyomyoma. Calcification typically occurs at least 6 months following UAE displaying a low signal intensity on $\mathrm{T} 1$ - and T2-weighted images along with blooming on GRE images.

Pre-embolization leiomyoma volume is the most important factor that determines its shrinkage following UAE rather than location and enhancement. Our imaging follow-up at 6 months showed a cumulative reduction of mean uterine volume by $30 \%$ at 6 months comparable to decrease of mean dominant fibroid volume by $44 \%$ where greatest reduction in myomatous volume was coupled with the smaller pre-UAE myomatous volume.

MR images should be interpreted in conjunction with clinical symptomology.

Post-UAE complications are rare. However, minor complications might occur like local hematoma at puncture site, transient pelvic pain, post-embolization syndrome adding to passage of necrotic material with or without delivery of leiomyoma. Femoral hematoma might be controlled with adequate compression and usually resolves spontaneously as long as it is localized with no vascular complication like pseudoaneurysm or fistula formation. Post-embolization syndrome is a constellateion of common findings that are experienced to a variable degree, encompassing pelvic pain and cramping, nausea and vomiting, low-grade fever, adding to general malaise and customarily controlled conservatively with analgesics and acetaminophen ${ }^{(26)}$.

Submucosal myomas complicated with trans-cervical expulsion; being frequent as 
2.5\% might be associated with intolerable pelvic pain, recurrent bleeding, or infection. In majority of cases, infarcted leiomyoma distends the endometrial canal and migrates toward the cervix. Occasionally, dilatation of the internal os and spontaneous passage may occur, depending on the size of infarcted leiomyoma. Nonetheless, larger leiomyomas might require hysteroscopic resection ${ }^{(27)}$.

Potential major complications include non-target inadvertent embolization and infectious complications. The former might result in premature menopause (ovarian dysfunction) and bladder necrosis; however, precise pre-procedural planning and preembolization comprehensive angiography greatly reduce this risk.

In our study, complications were very rare with no detectable post-procedural acute major complications which parallel metaanalysis of Torre et al. ${ }^{(\boldsymbol{( 6 )}}$. We had 5 minor complications, two of them experienced intolerable persistent pelvic pain necessitating night admission, however it was controlled afterwards by potent analgesics. One patient had postembolization syndrome but was resolved one week after; aided by analgesics and IM antibiotics. Efficient compression was enough to obviate complications following localized femoral hematoma in two patients.

\section{Conclusion:}

Although limited by small sample size however we might conclude that UAE is associated with considerable cumulative reduction of leiomyoma volume that parallels patients' symptomatic amelioration and psychological satisfaction and should be considered among first lines of management of symptomatic leiomyoma particularly in child-bearing age.

\section{REFERENCES:}

1. Toda A., Sawada K., Osuga K., Maeda N., et al. (2015): Tubo-Ovarian Abscess after Uterine Artery Embolization for Uterine Fibroid. Gynecol Obstet (Sunnyvale), 5:7
2. Toor S., Jaberi A., Macdonald D., McInnes M., et al. (2012): Complication Rates and Effectiveness of Uterine Artery Embolization in the Treatment of Symptomatic Leiomyomas: A Systematic Review and Meta-Analysis. AJR; 199:1153-1163.

3. Vilos G., Allaire C., Laberge P. and Leyland N (2015): The Management of Uterine Leiomyomas. J Obstet Gynaecol Can; 37(2):157-178.

4. Westcott M (2015): Uterine Fibroid Embolization IN Kipshidze N., Fareed J., Rosen R., Dangas G. and Serruys P. Urgent Interventional Therapies. John Wiley \& Sons, Ltd, $1^{\text {st }}$ edition: $474-484$

5. Martins J., Gaudenti D., Crespo F., Ganesh D. and Verma U (2016): Uncommon Complication of Uterine Artery Embolization: Expulsion of Infarcted Myoma and Uterine Sepsis. Case Reports in Obstetrics and Gynecology.

6. Torre A., Paillusson B., Fain V., Labauge P., et al. (2014): Uterine artery embolization for severe symptomatic fibroids: effects on fertility and symptoms. Human Reproduction; 29 (3): pp. 490-501.

7. Resnick N., Kim E., Patel R., Lookstein R., et al. (2014): Uterine Artery Embolization Using a Trans radial Approach: Initial Experience and Technique. $\mathbf{J}$ Vasc Interv Radiol; 25:443-447.

8. Van Overhagen H. and Reekers J. (2015): Uterine Artery Embolization for Symptomatic Leiomyomata. Cardiovasc Intervent Radiol; 38:536-542

9. Liang E., Brown B., Kirsop R., Stewart P., and Stuart A (2012): Efficacy of uterine artery embolization for treatment of symptomatic fibroids and adenomyosis An interim report on an Australian experience. Australian and New Zealand Journal of Obstetrics and Gynecology; 52: 106-112.

10. Ravina JH, Bouret JM, Fried D, et al. (1995): Value of preoperative embolization of uterine fibroma: report of multicenter series of 31 cases. Contracept Fertil Sex; 23:45-49. 
11. Mitty HA. (2010): Management of Obstetrical and Fibroid-Related Bleeding. Techniques in Vascular and Interventional Radiology; 3: 116-123.

12. Buttram VC and Reiter RC. (2009): Uterine leiomyomata etiology, symptomatology and management. Fertil Setril; 36:433-445.

13. Brunereau L, Herbreteau D, Gallas S, et al., (2009): Uterine artery embolization in the primary treatment of uterine leiomyomas: Technical features and prospective follow up with clinical and sonographic examinations in 58 patients. AJR; 175:1267-1272.

14. Levy S. and Sipes JB. (2010): Experimental internal iliac artery embolization: evaluation of low viscosity silicone rubber, isobutyl 2cyanacrylate, and carbon microspheres. Radiology; 129:51-58.

15. Marshburn PB, Matthews ML, Hurst BS (2006): Uterine artery embolization as a treatment option for uterine myomas. Obstetrics and Gynecology Clinics; 33(1):125-44.

16. Malone C., Banerjee A., Alley M., Vasanawala S., et al. (2018): Pelvic Blood Flow Predicts Fibroid Volume and Embolic Required for Uterine Fibroid Embolization: A Pilot Study With 4D Flow MR Angiography. AJR; 210: 1-12.

17. Liapi E., Kamel I. and Bluemke D (2005): Assessment of Response of Uterine Fibroids and Myometrium to Embolization Using Diffusion-Weighted Echo-planar MR Imaging. Comput Assist Tomogr; 29:8386.

18. Orazio T., Nicoletta C., Stefano M., Ugo L., Stefania T. and Claudia S (2015): Role of MRI for Evaluation of Uterine Giant Myoma: Case Report. International Journal of Medical Imaging; 3(3): 59-62.

19. Spielmann AL, Keogh C, Forster BB, Martin ML, Machan LS (2006): Comparison of MRI and sonography in the preliminary evaluation for fibroid embolization. American Journal of Roentgenology; 187(6):1499-504.

20. Das R., Gonsalves M., Vlahos I., Manyonda I., Belli A., et al. (2013): MRI Assessment of Uterine Artery Patency and Fibroid Infarction Rates 6 Months after Uterine Artery Embolization with Non-spherical Polyvinyl Alcohol. Cardiovasc Intervent Radiol.

21. Cura M., Cura A. and Bugnone A (2009): Role of Magnetic Resonance Imaging in Patient Selection for Uterine Artery Embolization. Acta Radiologica; 11051113.

22. Verma S., Gonsalves C., Baltarowich O., Mitchell D., et al. (2010): Spectrum of imaging findings on MRI and CT after uterine artery embolization. Abdom Imaging; 35:118-128.

23. Zhang J, Zou B, Wang K (2018): Spontaneous expulsion of a huge cervical leiomyoma from the vagina after cesarean: a case report with literature review. Medicine; 97(33): 50-55.

24. Deshmukh S., Gonsalves C., Guglielmo F. and Mitchell D (2012): Role of MR Imaging of Uterine Leiomyomas before and after Embolization. Radio graphics; 32: E251- E281.

25. Deipolyi A (2017): MRI enhancement predicting fibroid volume reduction to uterine artery embolization. Front Womens Healt; 2(2): 1-3.

26. Mutiso SK, Oindi FM, Hacking N, Obura T (2018): Uterine necrosis after uterine artery embolization for symptomatic fibroids. Case Reports in Obstetrics and Gynecology; 18: 50-55.

27. Ochmanek E, Brown MA, Rochon PJ (2019): Management of Complications in IR: Fibroid Expulsion after Uterine Artery Embolization. InSeminars in interventional radiology (Vol. 36, No. 2, p. 126). Thieme Medical Publishers. 
إنصمام الثريان الرحمي عن طريق القسطرة التدخلية كثقية فعالة قائمة بذاتها في علاج أعراض ألمات أورام الرحم الليفية

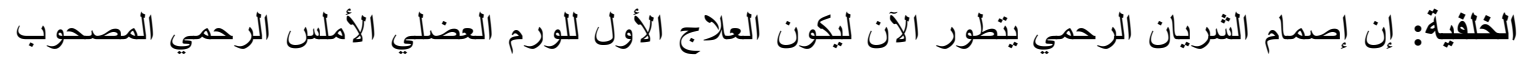

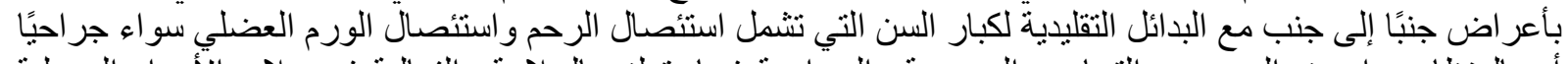

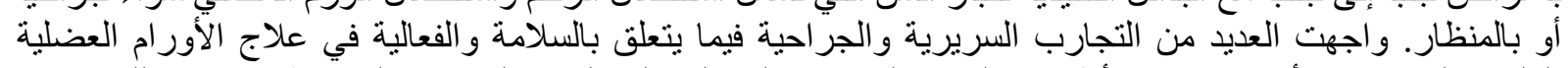

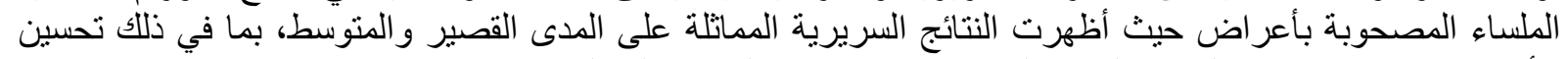

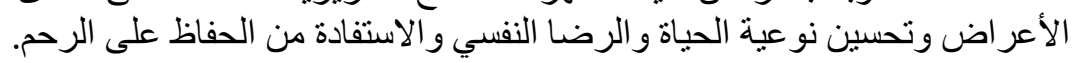

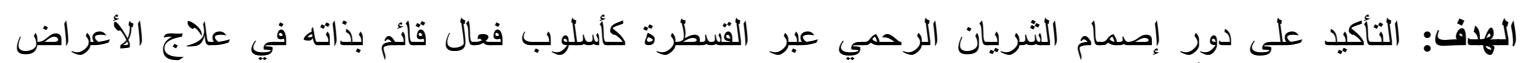
المنسوبة إلى الورم العضلي الأملس الرحمي.

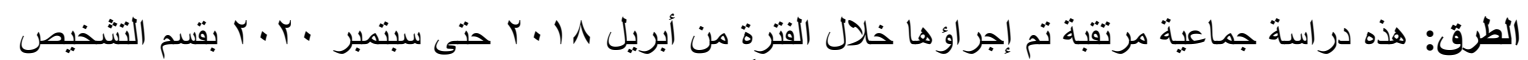

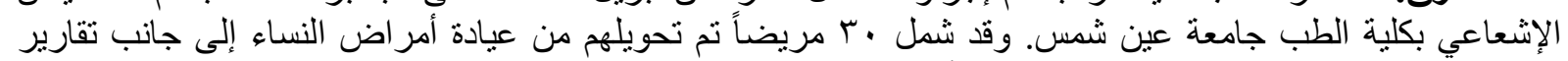

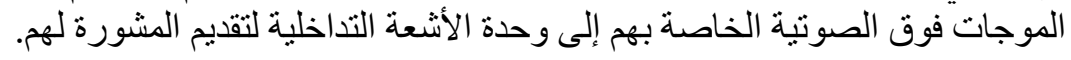

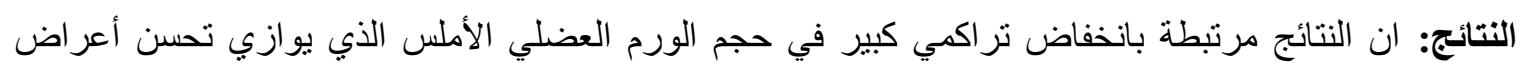

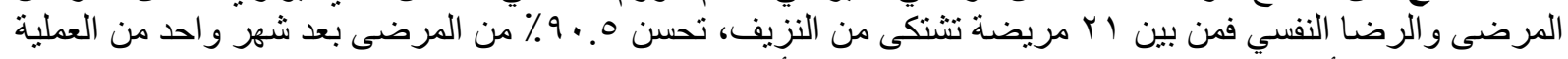

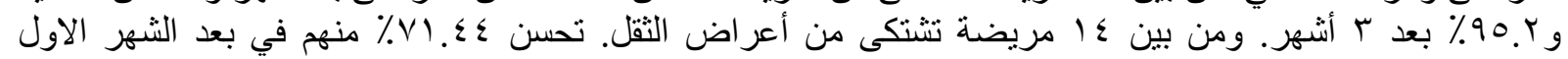

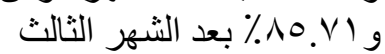

الخلاصة: يجب اعنبار انصمام الثريان الرحمى من بين الخطوط الأولى لإدارة الورم العضلي الأملس المصحوب بأعر اض خاصة في سن الإنجاب. 